\title{
The crystal structure determination of an organic dye with triclinic symmetry by electron diffraction
}

\author{
S. Plana-Ruiz ${ }^{1,2}$, Y. Krysiak ${ }^{3}$, J. Portillo ${ }^{4}$, E. Alig ${ }^{5}$, S. Estradé ${ }^{1}$, F. Peiró ${ }^{1}$, U. Kolb ${ }^{2,6}$
}

${ }^{1}$ LENS, MIND/IN2UB, Universitat de Barcelona, Martí i Franquès 1, 08028 Barcelona, Catalonia, ${ }^{2}$ Institut für Angewandte Geowissenschaften, Technische Universität Darmstadt, Schnittspahnstrasse 9, 64287 Darmstadt, Germany, ${ }^{3}$ Fyzikální Ústav, Akademie vĕd České Republiky, Cukrovarnická 10/112, 16200 Prague, Czech Republic, ${ }^{4}$ NanoMegas SPRL, Blvd Edmond Machtens 79, B-1080 Brussels, Belgium, ${ }^{5}$ Institut für Anorganische Chemie und Analytische Chemie, Goethe-Universität Frankfurt, 60438 Frankfurt am Main, Germany, ${ }^{6}$ Institut für Anorganische Chemie und Analytische Chemie, Johannes Gutenberg-Universität Mainz, Duesbergweg 10-14, 55128 Mainz, Germany.

sergiplana@hotmail.com /kolb@uni-mainz.de

3D Electron Diffraction (3D ED) has reached a point where it has become a routine technique for single-crystal diffraction studies at the nanometre scale [1]. Recently, some acquisition softwares have been developed with the aim of automatization and universal application [2]. One of them is the Fast and Automated Diffraction Tomography (Fast-ADT) [3]. This acquisition module is based on two tilt scans of the goniometric stage over the desired tilt range; the first one to monitor the crystal displacement with respect to the tilt angle in order to interpolate the necessary electron beam shifts, and the second one to acquire the diffraction patterns while automatically following the crystal. This procedure allows reliable diffraction acquisitions for crystals down to 20 nm provided that the stage has been aligned for tomography experiments and the holder is kept in good mechanical conditions. Fast-ADT can work in both TEM and STEM mode, but STEM is preferred mainly because of its low dose to acquire scanned images and its clear visualization of tiny or layered crystals in such conditions [4]. Another difference to other 3D ED routines is the use of Nano-Beam Electron Diffraction (NBED) instead of selected area electron diffraction. The combination of Fast-ADT and NBED enables several approaches focused on the optimization of 3D ED experiments, such as the shift of the beam at different positions of the same crystal or different crystals during diffraction pattern acquisitions. This versatility is beneficial as it gives the needed flexibility to study beam sensitive specimens even with post TEM column charged-coupled devices.

As an example, Fast-ADT was used to acquire datasets from disperse red 1 (DRED1) crystals, an organic molecule that was recrystallized in toluene. The DRED1 dye is an azobenzene derivate that is well known for their photochromatic properties and large optical and electro-optic properties in various polymeric films. The processing of six Fast-ADT datasets with eADT [5] revealed a triclinic crystal system with unit-cell parameters of $\mathrm{a}=7.72 \AA, \mathrm{b}=11.14 \AA, \mathrm{c}=19.58 \AA, \alpha=73.8^{\circ}, \beta=83.0^{\circ}, \gamma=70.5^{\circ}$ and $\mathrm{V}=$ $1523.5 \AA^{3}$ and indicated four molecules per unit cell. Simulated annealing, implemented in Sir2014 [6], was used to solve the structure and the positions of the azobenzenes were found using both $P 1(\mathrm{Z}=4)$ and $P-1\left(\mathrm{Z}^{\prime}=2\right)$. The small difference between the structure solutions obtained from both space groups was taken as an indication that the crystal structure could be described in the centrosymmetric one. However, the correct orientation of the flexible side chains was more difficult to retrieve because of their high degree of freedom. For this reason, two datasets were merged to obtain a higher number of independent reflections ( $85 \%)$ while, at the same time, a profile fitting of the rocking curves was applied to enable a frame orientation refinement and an improved reflection intensity integration [7]. These processing tools allowed to solve $a b$ initio the new polymorph of DRED1 in $P$-1, directly revealing the 46 non-hydrogen independent atoms from the electrostatic potential map. Finally, the structure model was refined based on X-ray powder diffraction data using the Rietveld method and a subsequent dynamical refinement based on 3D ED datasets enabled the proper description of the H-bonds of the crystal structure.

[1] Gemmi, M., Mugnaioli, E., Gorelik, T.E., Kolb, U., Palatinus, L., Boullay, P., Hovmöller, S. \& Abrahams, J.P. (2019). ACS Cent. Sci., 5, 8, 13151329.

[2] Smeets, S., Wang, B., Cichocka, M.O., Ångström, J., \& Wan, W. (2018). Zenodo. 10.5281/zenodo.2026774

[3] Plana-Ruiz, S., Krysiak, Y., Portillo, J. Alig, E., Estradé, S., Peiró, F. \& Kolb, U. (2020). Ultramicroscopy. $211,112951$.

[4] Plana-Ruiz, S., Portillo, J., Estradé, S., Peiró, F., Kolb, U., \& Nicolopoulos, S. (2018). Ultramicroscopy. 193, $39-51$.

[5] Kolb, U., Krysiak, Y. \& Plana-Ruiz, S. (2019). Acta Cryst. B 75, 463-474.

[6] Burla, M.C., Caliandro, R., Carrozzini, B., Cascarano, G.L., Cuocci, C., Giacovazzo, C., Mallamo, M., Mazzone, A., \& Polidori, G. (2015), J. Appl. Cryst. 48, 306-309.

[7] Palatinus, L, Brázda, P., Jelínek, M., Hrdá, J., Steciuk, G. \& Klementová M. (2019), Acta Cryst. B 75, $512-522$.

Keywords: 3D electron diffraction; automated acquisition; organic crystals; ab initio crystal structure determination 